\title{
Efficient processing technique based on plasma optical spectroscopy for on-line welding quality monitoring
}

\author{
J. Mirapeix, A. Cobo, A.M. Cubillas, O.M. Conde, J.M. Lopez-Higuera \\ Photonics Engineering Group, Univ. de Cantabria, Avda. Los Castros s/n, 39005 Santander, Spain
}

\begin{abstract}
In this paper a new spectroscopic analysis technique is proposed for on-line welding quality monitoring. This approach is based on the estimation of the wavelength associated with the maximum intensity of the background signal (continuum) of the welding plasma spectra. It will be demonstrated that this parameter exhibits a clear correlation with the welding quality of the seams, as it also happens with the traditional spectroscopic approach based on the determination of the plasma electronic temperature, thus allowing an identification of the appearance of weld defects. This technique offers a relevant improvement in terms of computational performance, what enables to detect smaller defects within the seam.
\end{abstract}

Keywords: arc-welding, on-line monitoring, plasma optical spectroscopy, continuum

\section{INTRODUCTION}

On-line quality monitoring for both arc and laser welding is an active area of research nowadays [1,2], as these processes are extensively used in key industrial areas such as aeronautics [3] or nuclear [4]. Among the different techniques that have been proposed, plasma optical spectroscopy has proved to be a promising approach, exhibiting as advantages the possibility of a robust analysis by means of the different atomic species participating within the plasma and the immunity of optical fibre sensors to electromagnetic interferences (of special interest in arc-welding scenarios), among others.

The spectroscopic analysis is typically based on the determination of the plasma electronic temperature, $T_{e}$, which is known to exhibit a correlation with the resulting welding quality [5,6]. A simplified expression involving only two emission lines is commonly employed to perform this task, allowing an improvement in the computational performance of the whole system. However, these emission lines have to be carefully selected, and issues regarding the identification of the lines may give rise to ambiguous results.

The identification of the plasma emission lines is based on the association to an initially unknown emission line of its corresponding chemical species and ionization stage. A possible solution to this problem lies in the use of a local database with the required spectroscopic information of the elements participating in the plasma. However, the typical limitations in the spectral resolution of low-cost CCD spectrometers (typically used in industrial scenarios) may be a major issue in terms of reaching an unambiguous line identification. Although some techniques can be applied to mitigate this situation, there is always a limitation in the selection of the emission lines, as it should be guaranteed that the difference between their upper level energies exceeds an established threshold [7]. With this scenario, it would be desirable to avoid the necessity of employing the identification stage, what could also lead to an improvement in the computational performance of the monitoring system.

A new spectroscopic analysis technique avoiding the use of the emission line identification stage is proposed in this paper. This new solution is based on the analysis of the plasma background (continuum) radiation. It will be demonstrated that there is a correlation between the wavelength associated with the maximum intensity of this signal and the quality of the associated seams.

Optical Sensors 2008, edited by Francis Berghmans, Anna Grazia Mignani, Antonello Cutolo, Patrick P. Meyrueis, Thomas P. Pearsall, Proc. of SPIE Vol. 7003, 70030Q, (2008) · 2277-786X/08/\$18 · doi: 10.1117/12.781055 


\section{TRADITIONAL SPECTROSCOPIC MONITORING PARAMETERS}

The traditional spectroscopic parameter used for on-line monitoring is the plasma electronic temperature $T_{e}$, which can be accurately determined by means of the Boltzmann-plot method. It can be derived from the expression relating the intensity of a given emission line to the population density of the upper level

$$
I_{m n}=N_{m} A_{m n} h \gamma_{m n},
$$

and the Boltzmann equation

$$
\ln \left(\frac{I_{m n} \lambda_{m n}}{A_{m n} g_{m}}\right)=\ln \left(\frac{h c N}{Z}\right)-\frac{E_{m}}{k T_{e}}
$$

where $I$ is the emission line intensity (induced by a transition from state $m$ to $n$ ), $\gamma_{m n}$ is the frequency associated with the emission line, $\lambda$ the wavelength, $A$ the transition probability, $g$ the statistical weight, $h$ the Planck's constant, $c$ the light velocity, $N$ the population density of the state $m, Z$ the partition function, $E_{m}$ the upper level energy and $k$ the Boltzmann constant. $T_{e}$ can be obtained from the representation of the left-hand side of equation (1) versus the upper level energy of each chosen emission line. By using several lines (of the same element in the same ionization stage), the resulting line will have a slope inversely proportional to $T_{e}$. Although the value of $T_{e}$ is more accurate with this method [8], the computational cost implied is significant, especially when real-time analysis is needed. Not only several lines have to be considered, but also a linear regression has to be performed to calculate $T_{e}$.

Although with less accurate results, the most common expression to estimate $T_{e}$ for welding monitoring is

$$
T_{e}=\frac{E_{m}(2)-E_{m}(1)}{k \ln \left[\frac{I(1) A(2) g_{m}(2) \lambda(1)}{I(2) A(1) g_{m}(1) \lambda(2)}\right]},
$$

given that only two lines are involved in the calculations, therefore reducing the computational cost. For the particular case of arc-welding, equation (3) varies, including in the logarithm of the denominator the quotient between the emission line upper level energies [9]

$$
T_{e}=\frac{E_{m}(2)-E_{m}(1)}{k \ln \left[\frac{E_{m}(1) I(1) A(2) g_{m}(2) \lambda(1)}{E_{m}(2) I(2) A(1) g_{m}(1) \lambda(2)}\right]} .
$$

A different approach may be also employed to determine $T_{e}$, where only a single emission line needs to be used. It is commonly referred to as the line-to-continuum ratio method [19], and in this case $T_{e}$ is calculated using the relation between a single emission line intensity (integrated over the line profile) $\varepsilon_{l}$ and the intensity of the adjacent background radiation (non-integrated) $I_{c}$

$$
\frac{\varepsilon_{l}}{I_{c}}(\lambda)=2.0052 \times 10^{-5} \frac{A_{m n} g_{m}}{Z_{i}} \frac{1}{T_{e} \xi} \exp \left(\frac{E_{i}-E_{m}}{k T_{e}}\right) \frac{\lambda}{\Delta \lambda},
$$

where $Z_{i}$ is the ion partition function, $\zeta$ the free-bound continuum correction, $E_{i}$ the ionization potential and $\Delta \lambda$ the wavelength bandwidth. Although the calculation of $T_{e}$ is not straightforward and an iterative method is required, the ratio $\varepsilon_{l} / I_{c}$ can be directly used as the monitoring signal, given that there is a direct relation between this quotient and $T_{e}$.

Equations (2) to (5) can be used when the plasma formed during the welding process is assumed to be both in local thermodynamic equilibrium (LTE) [8], and optically thin [9]. These assumptions are traditionally assumed to be valid for arc-welding plasmas, but it should be experimentally explored whether the plasma emission lines involved in the calculations are free from self-absorption [8]. 
It is worth noting that the line-to-continuum solution has not been typically used for on-line monitoring purposes. However, it exhibits a great potential in terms of computational performance, what led us to explore its feasibility. As we experimentally studied it, we found out that plasma continuum, in particular the wavelength associated with its maximum intensity, could be also a monitoring parameter of potential interest.

\section{ANALYSIS OF THE PLASMA CONTINUUM}

Figure 1 presents a possible processing scheme that can be used to determine $T_{e}$ with equations (2) to (4). Some stages need to be considered, for example a subtraction of the plasma background signal, a detection and modelling of the emission lines exceeding a particular intensity threshold, and, mainly, a proper line identification.

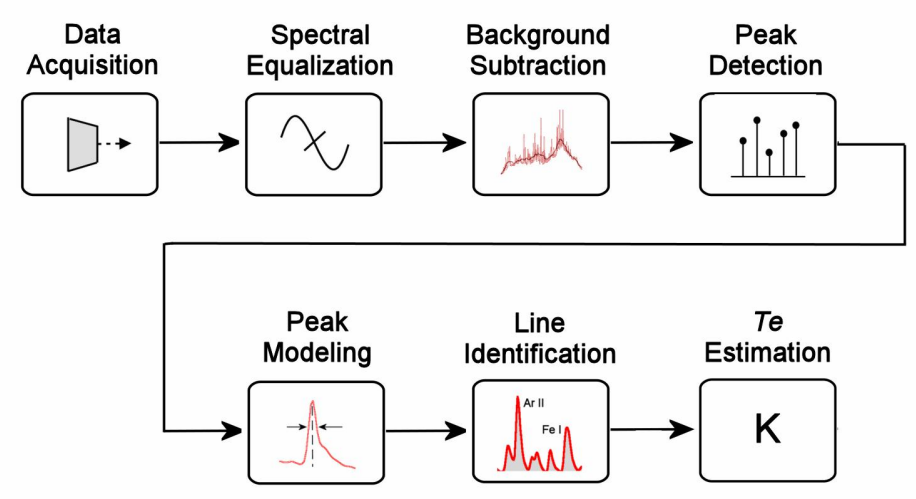

Fig. 1. Proposed processing scheme for on-line weld quality monitoring based on the estimation of the plasma electronic temperature.

As explained in Section 1, the identification of the lines implies a certain computational cost, as a search in a local database with the spectroscopic information of the species involved has to be performed. In fact, depending on the spectral resolution of the chosen spectrometer, additional steps should be also considered to obtain an accurate estimation of the emission line central wavelength [10], which is the key parameter in this process. The local database mentioned above can be designed with the information provided by the NIST atomic spectra database [11].

To accomplish the subtraction of the plasma background emission it is necessary to perform an initial estimation of this signal. Although there are different alternatives, like the use of a smoothing algorithm [10], we decided to estimate the continuum by employing a low-pass filter. Once a proper cut-off frequency is selected, the contribution of the plasma emission lines disappeared, and only the plasma continuum remains. In addition, this signal was also modelled as a blackbody radiation following the Planck function:

$$
B_{\lambda}(T)=\frac{2 h c^{2}}{\lambda^{5}} \frac{1}{\left(e^{\frac{h c}{k T \lambda}}-1\right)} .
$$

In equation (6) $T$ is the temperature obtained by means of the Wien's displacement law, which relates the wavelength associated with the maximum intensity of a blackbody radiation $\lambda_{\max }$ to its temperature. 
An example of this procedure is presented in figure 2, where the plasma continuum for two different welding spectra has been obtained. Figure 2(a) shows a plasma spectrum captured during an experimental welding test developed in the laboratory (the experimental setup will be detailed in Section 4). It can be observed that the spectral region considered covers from 300 to approximately $520 \mathrm{~nm}$. The plasma continuum (dotted line) and the resulting welding spectrum after the background removal are also depicted. A different welding spectrum can be observed in figure 2(b), where the same process has been considered to obtain the plasma continuum. The dissimilarities between both spectra are due to the different input optics, material to be welded and geometry of the welding process considered. While in the laboratory the plasma radiation was recorded by means of an optical fiber attached to an optical collimator, during the field tests a cosine corrector was employed to provide a light collection over $180^{\circ}$. In addition, the weld head used in the orbital arcwelding process in the field tests provides a completely different environment in comparison with the input optics disposition in the laboratory. For example, the angle formed by the normal to the input optics and the plasma axis differs among both approaches. Obviously, the different materials considered (AISI-304 in the laboratory and INCONEL-690 in the field tests) will also give rise to different spectra. Finally, the protection gasses were also different, as Ar was used in the laboratory and a mixture of $\operatorname{Ar}(40 \%)$ and $\mathrm{He}(60 \%)$ during the field tests.

\section{EXPERIMENTAL ISSUES}

The experimental tests developed in the laboratory were carried out by means of a TIG (Tungsten Inert Gas) welding system formed by a TIG power source (Kemppi Mastertig 2200) and a conventional TIG torch. AISI-304 stainless steel plates were attached to a positioning system and Argon was used as the required protection gas. The input optics of the monitoring system is based on a single optical fiber attached to a CCD spectrometer (Ocean Optics USB2000). As already mentioned at the end of Section 2, we initially explored the feasibility of the line-to-continuum method for online welding quality monitoring. Despite the significant computational cost reduction and the simplicity of the approach, we found out that the resulting profile, obtained as the quotient between the chosen emission line intensity and its associated background radiation, was highly dependent of the spectral band considered. This situation may give rise to false alarms or undetected defects, what is obviously highly undesirable in this context. This issue is illustrated by means of figure 3, where a seam with two subtle discontinuities is presented. The same experimental test was already studied in [10], where the difficulty of establishing a proper identification of the first defect at $\mathrm{x} \approx 4.5 \mathrm{~cm}$ was discussed in detail. Three different spectral bands have been selected for figures 3 (b), (c) and (d). It can be appreciated that both figures 3(c) and (d) exhibit relevant perturbations at the defect locations. However, the resulting profiles are somewhat unstable, what would make it difficult to select the threshold to raise and alarm. On the other hand, the analysis performed with the spectral band of figure 3(b) does not provide a clear correlation to the seam quality events.

A different experimental test is studied in figure 4 . In this case, both $\lambda_{\max }$ and $T_{e}$ profiles have been included to allow a direct comparison among these techniques. Figure 4(a) presents a seam with a clear defect at $\mathrm{x} \approx 5 \mathrm{~cm}$, which was produced by performing a reduction on the protection gas flow during approximately $0.5 \mathrm{~s}$. The correlation between this defect an the $\lambda_{\max }$ profile of figure 4(b) is obvious, as this signal has a mean value around $485 \mathrm{~nm}$ that significantly varies associated with the gas cut. As expected, there is also a direct correlation between figures 4(b) and (c). In the latter, the $T_{e}$ profile of the so-called traditional spectroscopic approach has been depicted.

The proposed technique has also been checked during field tests of orbital arc-welding process on weld coupons [12]. Not only the differences between the processes, but also the application in a real industrial scenario make this results worthwhile. To check the performance of the technique, a porosity was intentionally produced during the process by applying a gel on the tube-to-tubesheet interface [12]. This porosity can be appreciated in figure 5(a), while the rest of the seam dos not exhibit any other visible anomaly. We calculated the associated $\lambda_{\max }$ profile (in this case related to the welding time of the process), and we found out that a clear perturbation appears associated with the porosity (figure 4(b)). This result suggests that this technique could be a valid alternative to the traditional spectroscopic approach for online welding quality monitoring. 


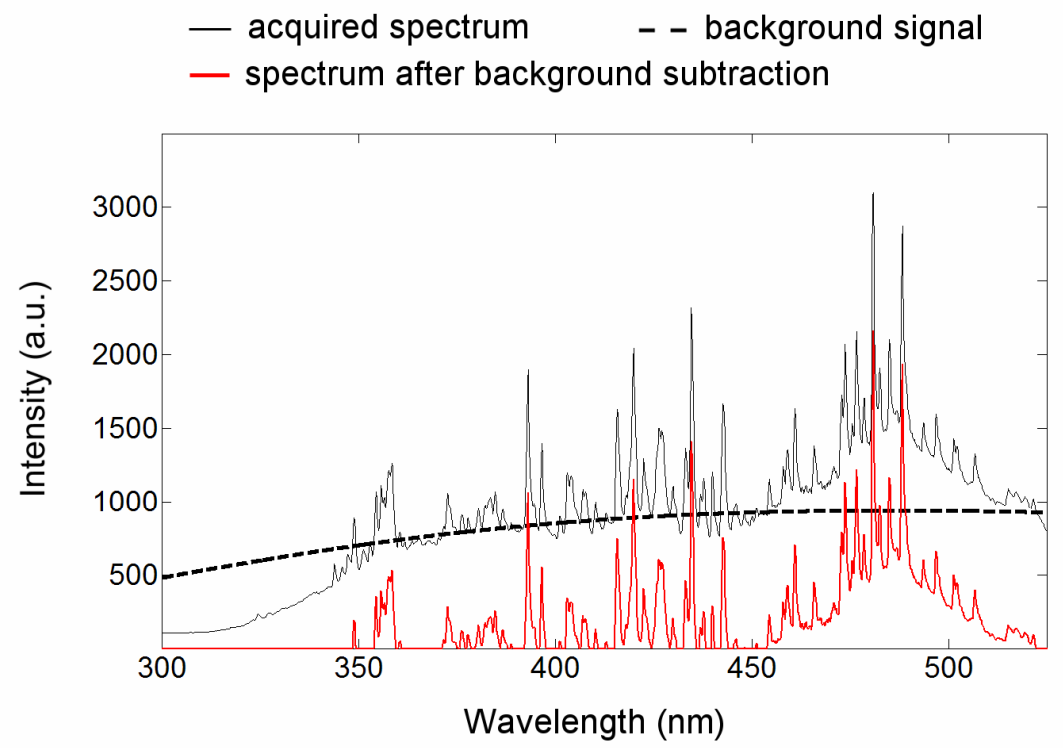

a) Experimental spectrum background signal

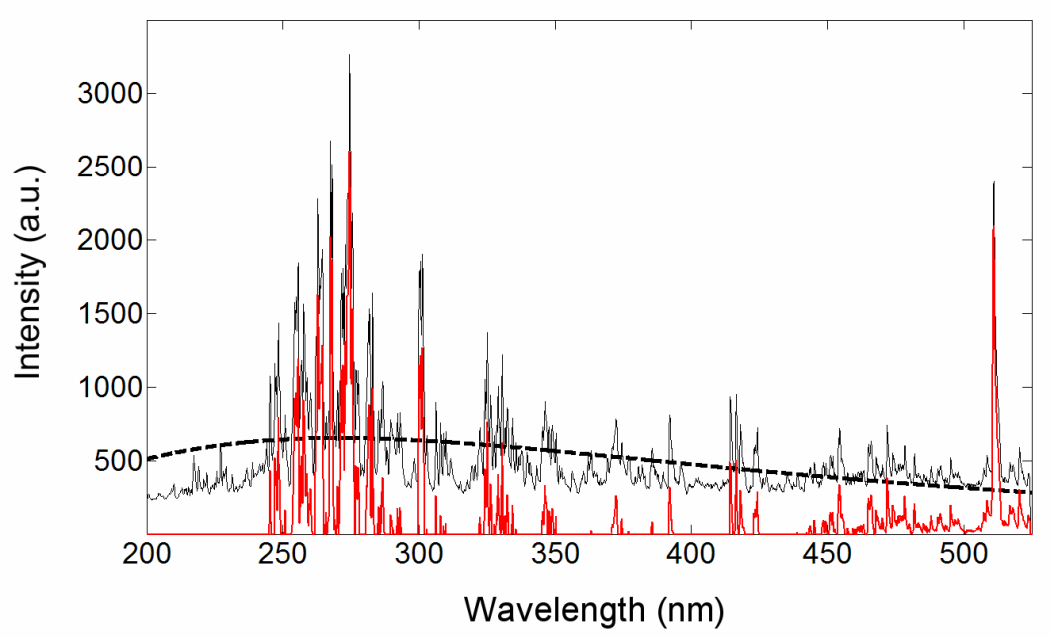

b) Field test spectrum background signal

Fig. 2. Experimental (a) and field test (b) spectra and their resulting background signal after the filtering and modeling process. 


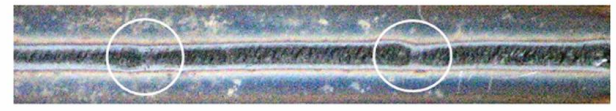

(a)

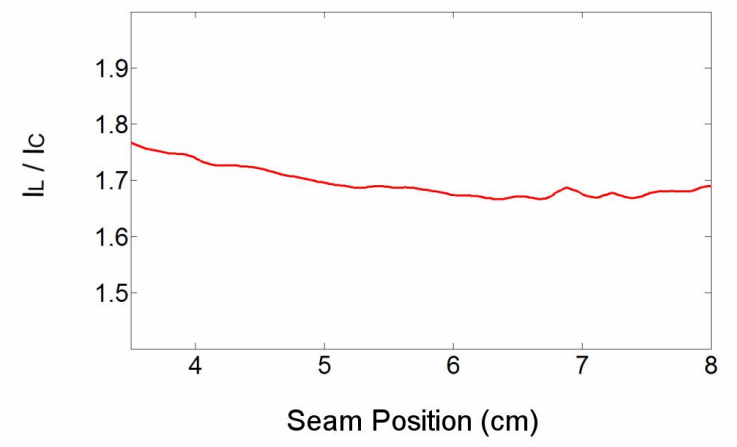

(b)

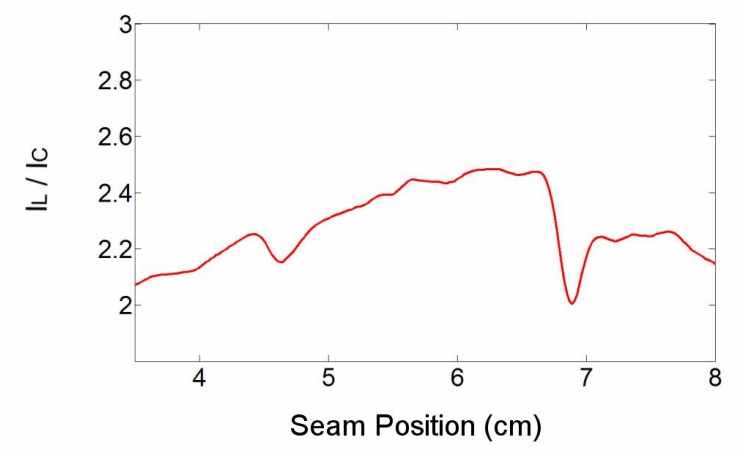

(c)

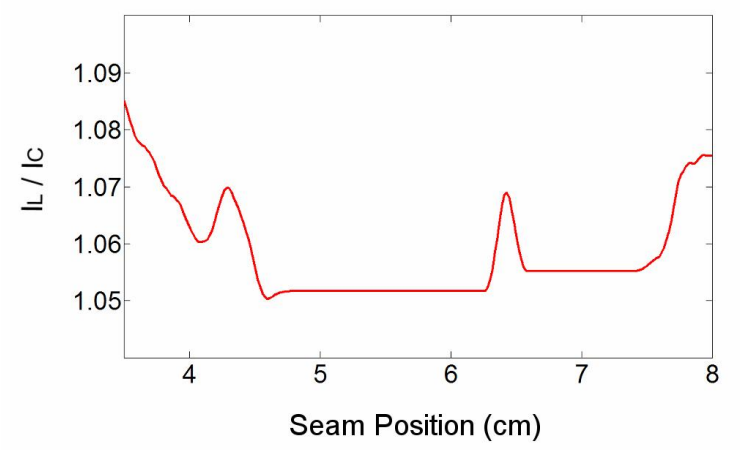

(d)

Fig. 3. Line-to-continuum method applied to the detection of seam defects: (a) weld seam with discontinuities; (b) line-tocontinuum signal $(472.991 \mathrm{~nm})$; (c) line-to-continuum signal $(482.743 \mathrm{~nm})$; (d) line-to-continuum signal $(377.97 \mathrm{~nm})$ 
(a)

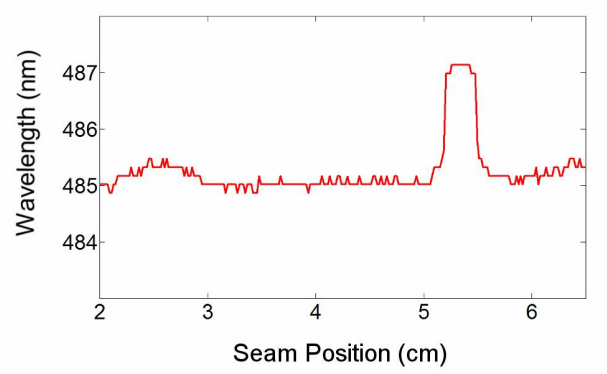

(b)

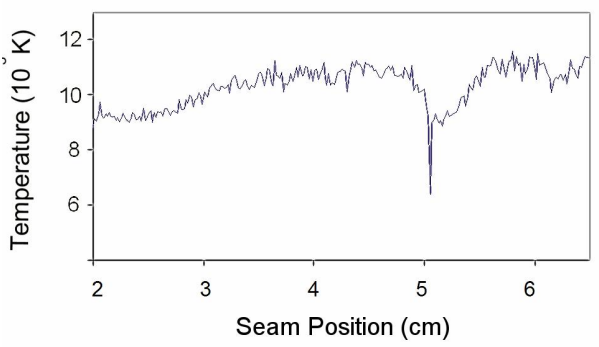

(c)

Fig. 4. Comparison of spectroscopic monitoring parameters: (a) weld seam with defect; (b) $\lambda_{\max }$ after continuum modeling via filtering; (c) $T_{e}$ profile

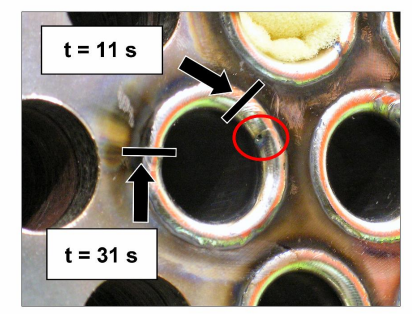

a) Welded tube with porosity

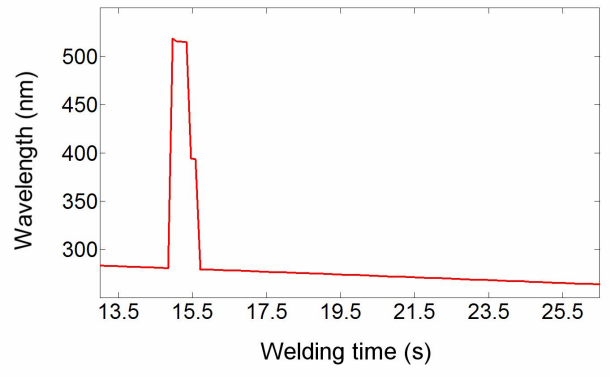

b) $\lambda_{c-m a x}$ profile (filtering)

Fig. 5. Correlation of the $\lambda_{\max }$ profile and porosity in a seam performed during the field tests 


\section{CONCLUSIONS}

A new spectroscopic approach based on the analysis of the plasma continuum for on-line welding quality monitoring has been presented in this paper. In our search for a new efficient alternative to the traditional spectroscopic solution focused on the estimation of the plasma electronic temperature, we initially explored the feasibility of the line-to-continuum method. This technique, which also allows a plasma temperature calculation, would imply a significant saving in the computational times involved in the analysis. However, our studies have led us to the conclusion that it is not reliable, at least with the simplifications that we have assumed in our approach. The dependence of the resulting monitoring signal on the chosen spectral band is a mayor issue to investigate, as well as the apparent instability of the profiles. On the contrary, the performance exhibited by the analysis of the plasma continuum is very promising, both in terms of computational and monitoring performance. The processing requirements needed to obtain the wavelength associated with the maximum intensity of the plasma continuum are very simple, and it has been demonstrated that there is a clear correlation between the quality of the seams and the proposed monitoring signal. Although this is an exploratory approach and more studies should be conducted, we believe that this technique could be effectively used in real industrial scenarios.

\section{ACKNOWLEDGEMENTS}

This work has been co-supported by the Spanish TEC'2005-08218-C02-02 and TEC'2007-67987-C02-01 projects. Authors want to thank S. Fernandez and J.J. Valdiande for their valuable help during the experimental tests, as well as the staff of ENSA (Equipos Nucleares S.A.), specially D. Solana, F. Linares and R. Cardoso.

\section{REFERENCES}

[1] Wu, C.S., Gao, J.Q. and Hu, J. K., "Real-time sensing and monitoring in robotic gas metal arc welding," Meas. Sci. Technol. 18, 303-310 (2007).

[2] Sibillano, T., Ancona, A., Berardi, V. and Lugara, P.M., "Real-time monitoring of laser welding by correlation analysis: The case of AA5083," Optics and Lasers in Engineering 45(10), 1005-1009 (2007).

[3] Bardin, F., Cobo, A., Lopez-Higuera, J.M., Collin, O., Aubry, P., Dubois, T., Hogstrom, M., Nylen, P., Jonsson, P., Jones, J.D.C. and Hand, D.P., "Optical techniques for real-time penetration monitoring for laser welding," Applied Optics 44(19), 3869-3876 (2005).

[4] Cobo, A., Mirapeix, J., Linares, F., Piney, J.A., Solana, D., and Lopez-Higuera, J.M., "Spectroscopic Sensor System for Quality Assurance of the Tube-To-Tubesheet Welding Process in Nuclear Steam Generators, " IEEE Sensors J. 7, 1219-24 (2007).

[5] Sforza, P. and de Blasiis, D., "On-line optical monitoring system for arc welding," NDT\&E Int. 35, 37-43 (2002).

[6] Ancona, A., Spagnolo, V., Lugara, P.M. and Ferrara, M., "Optical sensor for real-time monitoring of CO2 laser welding process," Appl. Opt. 40, 6019-6025 (2001).

[7] Lochte-Holtgreven, W., [Plasma Diagnostics], North-Holland Publishing Company, Amsterdam, 45-178 (1968).

[8] Griem, H.R., [Principles of Plasma Spectroscopy], Cambridge University Press, Cambridge (1997).

[9] Marotta, A., " Determination of axial thermal plasma temperatures without Abel inversion,", J. Phys. D: Appl. Phys. 27, 268-272 (1993).

[10] Mirapeix, J., Cobo, A., Conde, O.M., Jaúregui, C. and López-Higuera, J.M., "Fast algorithm for spectral processing with application to on-line welding quality assurance," Meas. Sci. Technol. 17(10), 2623-2629 (2006).

[11] National Institute for Standards and Technology (NIST) atomic spectra database (http://physics.nist.gov/cgibin/AtData/main_asd).

[12] Cobo, A., Mirapeix, J., Linares, F., Piney, J.A., Solana, D., and Lopez-Higuera, J.M., "Spectroscopic Sensor System for Quality Assurance of the Tube-To-Tubesheet Welding Process in Nuclear Steam Generators, " IEEE Sensors J. 7, 1219-24 (2007). 